\title{
We Have the Spaceship; But Where's the Start Button: Human Engineering Issues in the Age of Long Duration Space Exploration
}

\author{
George S. Hamilton \\ NASA \\ Christopher W. Adams \\ Raytheon
}

Copyright @ 2005 SAE International

\begin{abstract}
As long duration space exploration and habitation becomes more commonplace, a number of Human Engineering factors (Gravitational Adaptation, 2-D to 3-D Movement Adaptation, Design Form/Function, and Space Ergonomics to name a few) will become more pronounced. More research and development is needed in these areas or the explorers may find themselves in painful or dangerous situations.
\end{abstract}

\section{INTRODUCTION}

Human occupation of space is rife with dangers. Radiation, temperature extremes, lack of oxygen, and a huge fall back to the ground are just some of the issues that make space one of the most hostile environments imaginable. But, through all of that you never hear anyone say, "Oww, my back hurts." Well, not yet. As we move in the direction of long duration exploration and habitation in space and on other planetary bodies new human factor issues will throw themselves into the mix. And as we begin to commercialize space travel we won't be able to count on strong, well-trained bodies to be able to cope with those stressors. Some of these issues have the potential to cause great suffering, injury and even mission failure if not properly addressed before we take flight.

\section{MAIN SECTION}

GRAVITATIONAL ADAPTATION - NASA's The Vision for Space Exploration [NASA, 2004] outlines NASA's goal for a manned mission to Mars. In a possible reference mission to Mars crew members live and work in a cycle of 9 gravitational environments from launch to landing (excluding short duration launch and landing gforces). Terran, lunar, Martian, and micro-gravity environments, as well as exercise related "artificial gravity" environments, present a serious challenge to crew operations and safety. Although crew members have demonstrated the ability to adapt to different gravitational environments with no major issues (based on 6 months work), the minor issues present a risk. Delightful crew anecdotes about their mental gravitational shifts to micro-gravity when back on Earth, such as floating a coffee cup only to hear it shatter as it hits the floor a second later, or fighting the urge to float down the stairs are entertaining in the proper setting, but they may indicate a serious operational obstacle in manned missions to Mars. This anecdotal evidence suggests that crew members may lose their adaptation during menial tasks. The crew "forgetting" what gravitational environment they're operating in can lead to performance degradation, hardware damage, and personal injury.

Humans have exhibited a remarkable ability to adapt to micro-gravity and then instantaneously re-adapt to Earth's gravity with no gross ill effects. Crew members are able to walk off the shuttle after months in microgravity without wobbly knees, but they have been known to slip up in menial tasks.

The crew, once adapted, is able to perform many menial tasks, or tasks that don't require concentrated thought, without losing adaptation, such as floating in microgravity and walking in $1-\mathrm{g}$. This observation leads to the theory that there are constant environmental reinforcements that aid in maintaining adaptation. Gravitational pull on the body helps maintain an erect posture and pressure on the soles of the feet help in determining the proper force needed to stand; however, an action such as absent-mindedly determining a temporary stowage location for a drinking vessel does not have any cues to reinforce which mental path the decision making process should take.

Isolated actions like this can be misjudged by the crew. The wrong application of force could strain the hardware or crew muscles and tendons. The wrong translation 
method could result in crew injury. Having to consciously remember what gravitational environment the crew is operating in may use one of Miller's $7+/-2$ memory retention units. Emergency operations may be slowed. And existing mental errors may worsen. These issues become compounded when the crew must live and work in multiple environments. A Mars mission could require the crew to spend an ample amount of time training on Earth $(1-g)$, transferring in micro-gravity with temporary workout in artificial gravity (most likely between $1 / 12-\mathrm{g}$ and $1 / 8-g)$, spending time on the moon $(1 / 6-g)$, then to the transfer vehicle, then to Mars $(1 / 3-g)$, back to the transfer vehicle, another stop on the moon, one last trip in the transfer vehicle, and finally a landing on Earth where they'll live out their days.

Environmental cues may be an effective countermeasure to momentary adaptation loss. By providing each unique gravitational environment with its own sensory schema the crew would be constantly "reminded" of what gravity environment they are operating in. Visual, physical, auditory and olfactory cues could form a synergistic schema to differentiate Mars from the moon and aid crew operations and habitability, particularly if those cues become part of the background and do not serve as a distraction.

2-D TO 3-D MOVEMENT ADAPTATION - On Earth humans move mostly in two dimensions. That is, we're held to the Earth by gravity and typically only move on a plane. In a micro-gravity environment humans can adapt to 3-dimensional movement. Observation of the crew shows us that the switch usually takes place within 1 to 3 days of being introduced to that environment. However, no time frame is set, since adaptation seems to be a personal matter.

NASA operation planners already take this into account by easing the crew into complicated tasks. Allowing the crew time to learn to control their own body and manipulate objects in micro-gravity increases the mission success rate.

It has been observed that when the "switch" becomes complete the crew can move effortlessly, almost intuitively, using just the right application of force and moving in the most efficient manner. A typical signal to this change is when the crew stops re-orienting themselves. At first a crew member will normally turn in the direction they want to travel, head first, and then push off as if they are flying. After they adapt they just float to where they want to go without having to re-orient their bodies.

The crew also gains an understanding of how foreign bodies act. They no longer feel the need to strap down every item they are using to keep them from floating away. They will temporarily stow an item in mid air and then retrieve it when needed.

Understanding when and how this adaptation takes place is important. But what is also important is designing for this capability. Those who have only experienced 2dimensional motion, that is most of us, need to break away from our instinctual knowledge of body mechanics to design hardware for efficient micro/partial gravity environments. This is not an easy thing to accomplish. We have the knowledge of how the body moves ingrained in our brain since birth, literally. The first time you realize that humans don't squat in micro-gravity to examine the bottom shelf, but float upside down can serve as an eye opener or rude awakening, depending on the circumstances.

Ideally this problem would be solved by giving all those involved with space-flight hardware a weekend trip to the space station. Instead we must observe. We must observe crew operations, body mechanics and posturing in micro-gravity. And we must stay diligent in applying the findings from these observations. A large library of crew video and stills exist and should be consulted often.

EVERYTHING'S A HANDLE - There is an old automotive corollary that states, "When in need, everything's a hammer." This is used to illustrate that you can beat on something with any tool, no matter what its intended design was. Micro-gravity environments have a similar corollary. "When in need everything's a handle."

In January of 2004 International Space Station crew located a small air leak that had plagued them for weeks. The culprit was a small break in a vacuum jumper for the Destiny module's optical-quality window (figure 1). A vacuum jumper is a flexible hose used to help equalize air pressure across the window [Oberg, 2004 a].

The Russian Aviation and Space Agency stated they "believe the U-shaped hose near Destiny's main window was repeatedly bent by station crew members who used it to stabilize themselves while taking pictures out the window [Oberg, 2004 b]." NASA officials agreed. In other words, it was used as a handle. And not just by one person. The Itar-Tass news agency quoted one Russian official as saying, "The present crew is no more guilty than the preceding ones."

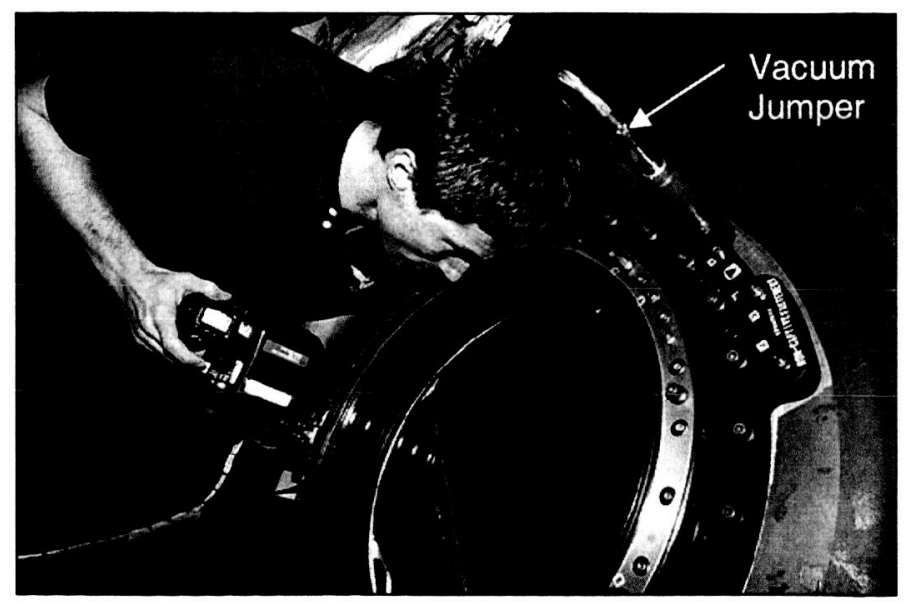

Figure 1 Destiny's optical quality window 
This just goes to show that: if it looks like a handle; if it feels like a handle; if it acts like a handle, then it is a handle. When placed in a micro-gravity environment the crew will automatically use whatever is in reach to aid them in translation, stability and body control. Providing appropriate crew restraints and mobility aids is the ideal solution, and a goal of the International Space Station program. However, if it looks like a handle, it is a handle, no matter how many "Do Not Grasp" labels are placed there.

The designer's adage, "Form Follows Function," has an often overlooked inverse, "Function Follows Form." The function of an item should not be misconstrued due to its form. In some cases this means that form cannot follow function. A form needs to communicate its intent. Barbed wire means don't touch. Soft, velvety pillows mean take a nap. A U-shaped semi-rigid hose next to the one thing that crew members will stare out of for hours on end means it was designed to fulfill its job in a straight forward manner without thought to its immediate surroundings or perception.

Designers need to be aware of this and design hardware with this in mind. Don't assume that the crew will only touch the areas that you designed to be touched. If there is an item that should never be grabbed, hide it. If you do have a handle or grasp area designed into the hardware, make sure it is logically placed for the crew's use (See above mentioned 2-D TO 3-D ADPTATION) and that it is more attractive as a handle than anything else on the hardware. Furthermore, if it is a grasp area, or looks like one, make sure the structure can take the abuse that repeated application of crew loads provides.

EXERCISE POSTURE - One of the means of bone and muscle mass preservation countermeasures on Space Station is the Treadmill with Vibration Isolation and Stabilization system, or TVIS, which is located in the Zvezda Service Module. The International Space Station benefits from the Russian Mir Space Station experience where the fixed treadmill was hard mounted to the structure, and all of Mir oscillated when the treadmill was in use, defeating the micro-gravity environment needed for some science experiments (figure 2).

TVIS uses a spring loaded waist \& shoulder harness to hold the crew on the treadmill and provide $66-100 \%$ of body weight to determine the intensity of the workout (figure 3 ). The running posture looks remarkably similar to $1-g$, and one wonders if that similarity will remain for a long duration space flight on the order of a two year round trip to Mars. Note the normal " $S$ " curve of the spine (figure 3 ), in contrast to the traditional notion of a resting Neutral Body Posture "C" curve (figure 4).

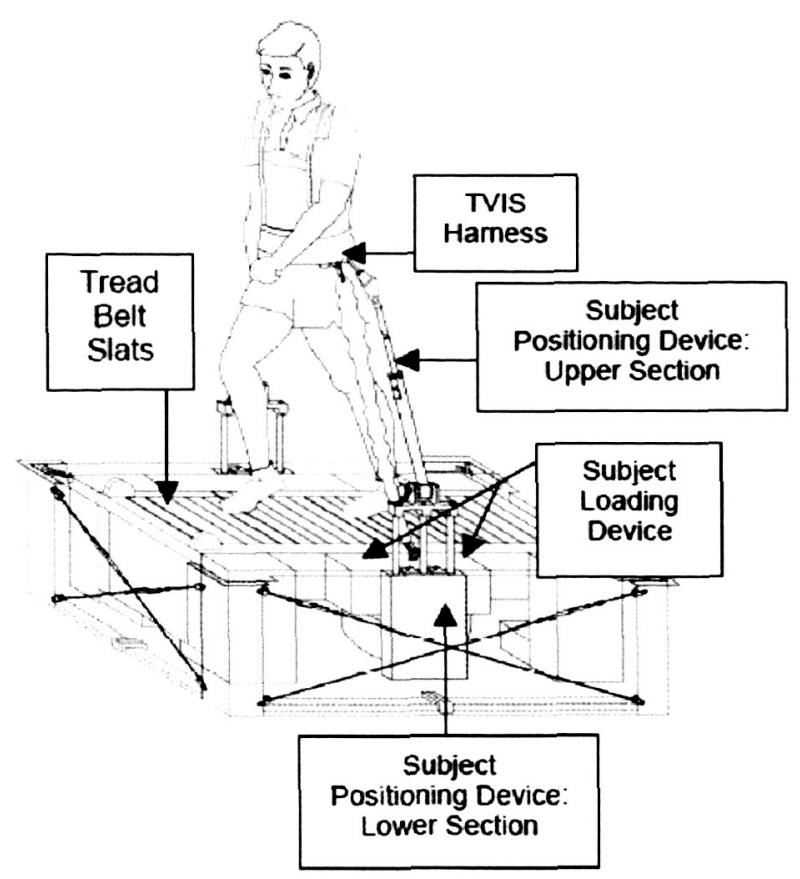

Figure 2 TVIS

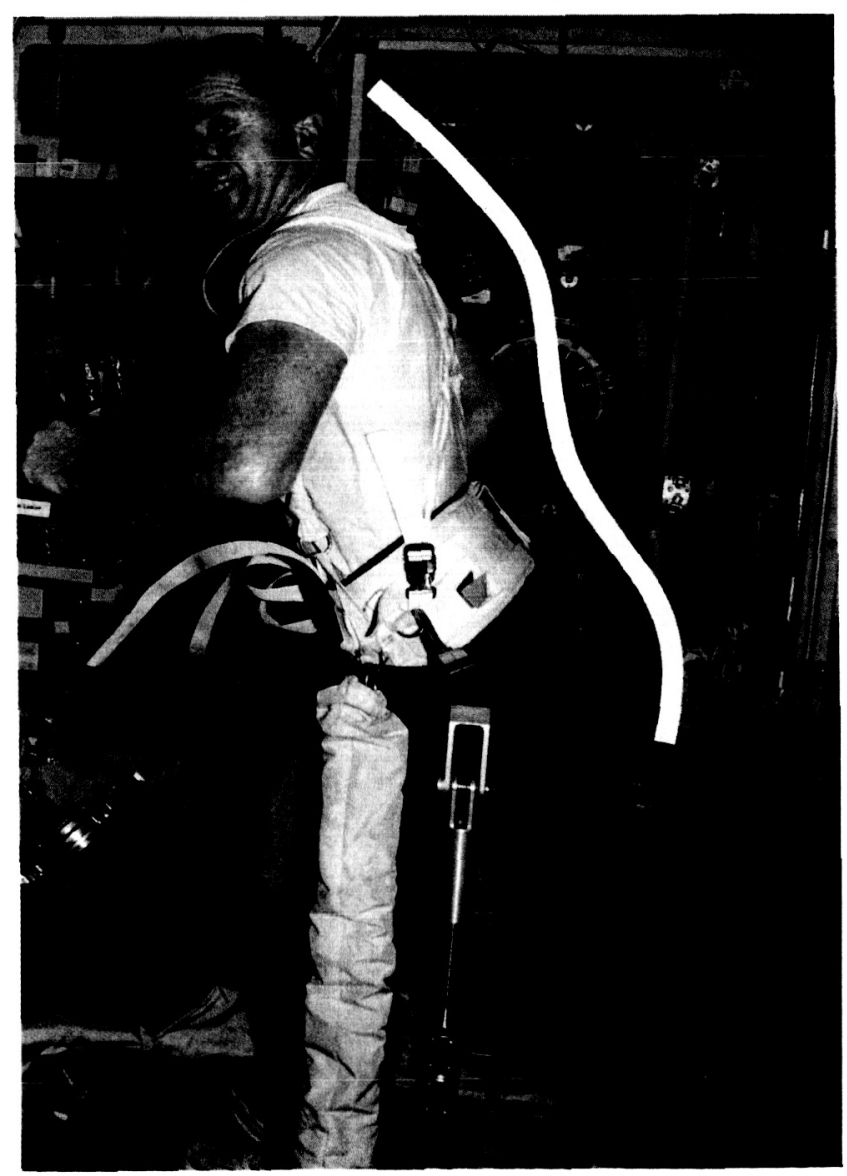

Figure 3 TVIS side view showing "S" shaped spine 


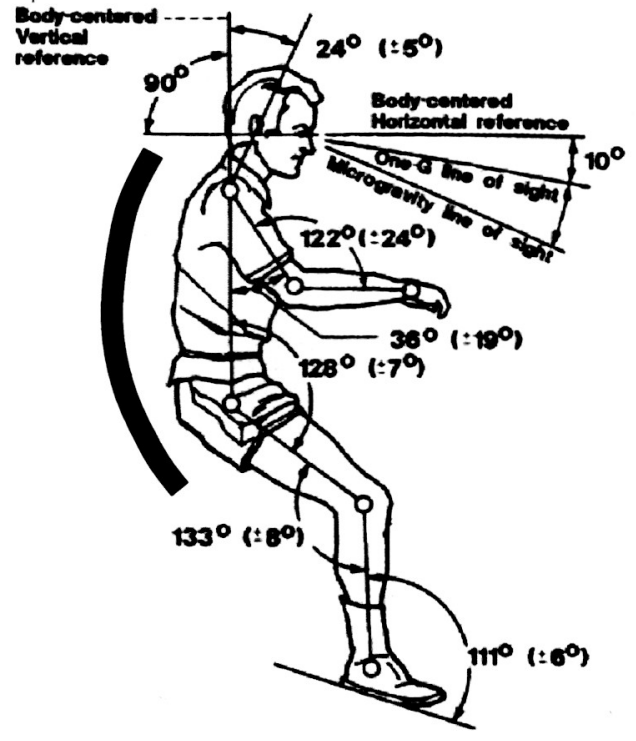

Figure 4 NBP from NASA-STD-3000, showing "C" shaped resting spine

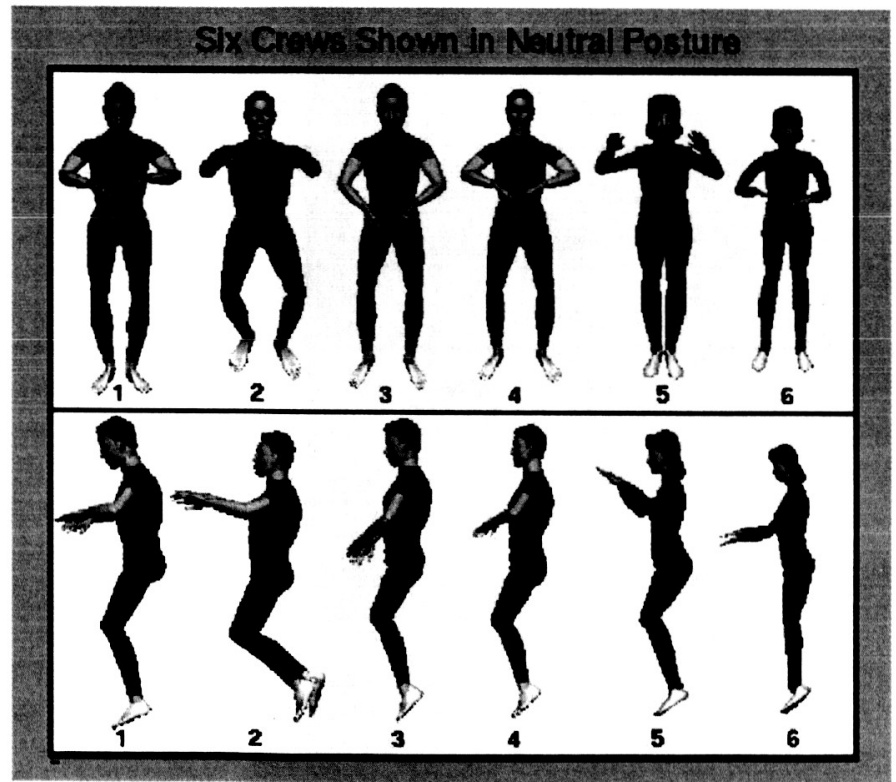

Figure 5 Recent NBP from STS 57

However recent work has called into question the Neutral Body Posture (NBP) as reported in reference six [Mount, 2003]. This study conducted in 2003 , suggests that the NBP varies significantly from the composite shown in figure 4, perhaps due to gender, age and athletic development (figure 5). This discovery may have significant impact on the posture used to design microgravity workstations. This result is not surprising as the small sample used for figure 4 consists of 12 pictures of three crew members who were short, young, slender, male, Caucasian, test pilots; a group rather unlike the crew members which fly today. What effect the deviation in NBP may have on the design or efficacy of treadmill or other micro-gravity countermeasures is unknown and fertile ground for research.
The classic Skylab NBP results from all opposing skeletal muscle groups in balance at equilibrium. Figure 5 shows that different individuals have different muscle balances, thereby deviating from the classic NBP. The good posture exhibited in figure 3 is also likely derived from running in 1-g. The question is how long that posture will hold up in micro-gravity or reduced gravity. A continuation of the STS-57 NBP study analyzing a crew member's NBP over long duration space flight may shed more light on the some issues. Identifying differences in the NBP from the beginning of a mission through the end of a mission may show changes in muscle balancing.

Doctors and fitness instructors say that proper posture is one of the keys to avoiding injury during exercise. A good exercise posture supports a healthy S-curve. A crew member's exercise posture has two detrimental effects placed on it: 1) the vast amount of time spent in the NBP with the related C-curve weakening the natural S-curve, and 2) the TVIS shoulder harness straps exerting downward pressure at the shoulders. The NBP is characterized by having all the muscles balanced at rest; however muscle loading will be different than in 1-g because of this and could alter the existing muscle balance. As for the harness, the pull of the straps cause the crew member to want to hunch over and roll their shoulders forward. Both of these factors may act to change a proper S-curve. And exercising with an "improper $S$ curve" posture in micro-gravity may cause injury.

Further consideration must be given to opening up of space exploration to the general populace. Currently all space travelers go through rigorous physical training and are in very good shape. That may not be the case in the near or medium term future.

Poor exercise posture could have a hazardous effect on a crew member's physical and mental well being during a trip to Mars, or an extended tour of duty on the International Space Station. The effects are currently only theorized since good muscle strength can usually combat the negative stimuli over the short term. But since we've theorized the problem, we went ahead and theorized some solutions. The use of a properly designed orthotic "S-curve support" during TVIS use or the design of a recumbent bicycle exercise device with lumbar support could both help alleviate the problems and strengthen the S-curve.

SPACE ERGONOMICS - Exploration is an innate part of the human spirit. And as such, colonization of heavenly bodies is a forgone conclusion. Also innate to the human spirit is to strive for efficiency, or a penchant for laziness. After all, they are two sides to the same coin. This has led to a rather useful science called "Ergonomics." But what is "Space Ergonomics?"

Answer: A set of design principles based on ergonomic research and evaluation as applied to the man-machine 
interfaces and interaction particular to micro-gravity (or partial gravity) environments.

Volumes of anthropometric data exist. Rooms full of micro-gravity operation observations and evaluations exist. Countless spacecraft and habitat designs exist. But very few space related human-machine interfaces were designed with usability as the primer. Most items start either in the imagination or with a technical solution. A systematic approach stemming from the operation and human-machine interface of the hardware is hardly ever used. However, when this approach is used the results are typically extremely successful. One such success story is the Payload Equipment Restraint System, or PERS (figure 6).

"The Payload Equipment Restraint System (PERS) assists the crew in restraining and transferring payload equipment and tools in order to maximize the crew time available for payload operations and to minimize the crew time required for handling of the equipment. The system is designed to aid the crew during all phases of payload installation, removal, and maintenance operations. It can be used to transport items from stowage locations to the worksite as well as temp stow those items at the worksite. Certain elements of the system can also be used as permanent stowage for payload equipment and tools."

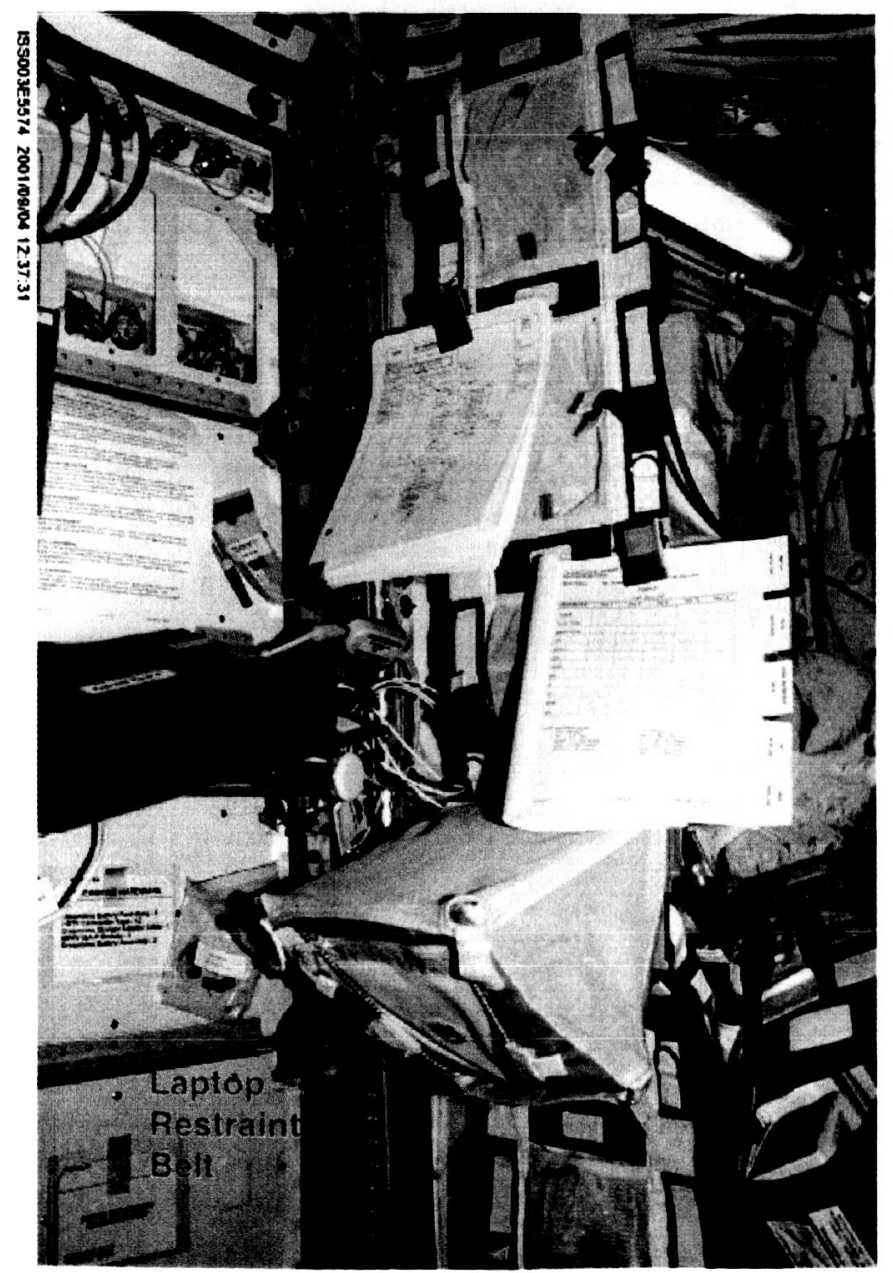

"PERS is a modular system consisting of five elements; the (1) Belly Pack, (2) Laptop Restraint Belt (LRB), (3) Tool Pages, (4) Single Strap, and (5) H-Strap. It's designed to work in concert with existing International Space Station (ISS) equipment such as the Restraints and Mobility Aides (R\&MA), Laptops, Cargo Transfer Bags (CTB), and the Inventory Management System (IMS) Bar Code Reader [NASA, 2001]."

PERS developed a set of design principles that took into account: the physical limitations of the crew in a microgravity environment; the operational needs of the equipment being interfaced with; the operational needs of the crew; and, an often overlooked item, the typical behavior and capabilities of the crew. PERS found overwhelming success aboard the International Space Station and has become a favorite tool of the crew due to its usability and adaptability. The design principles that governed the development of PERS have been used to contemplate and design various other restraints and mobility aids that mimic PERS' effectiveness and share the same interfaces the crew has grown to depend on.

Decades of study in ergonomics have resulted in an increase in most consumers' quality of life. And those in the field know there is still much more work to do. Both space and space ergonomics are new frontiers, with untapped dangers and possibilities waiting in the dark. PERS and other programs like it have started chipping away at the mountain of work needing to be done. It's time to pick up an axe, and really start working to better the quality of life of our future brave explorers and colonizers of the worlds still waiting for us.

\section{CONCLUSION}

The U.S. vision of space exploration brings with it great excitement and opportunity. It also brings in a new load of problems to be solved. Ensuring the crew will stay within a given gravitational adaptation; educating designers about the crew's 3-D movement adaptation and common human factors issues and needs, such as everything being used as a handle; providing the crew with the best designed exercise equipment to ensure they maintain a strong and healthy body; and systematically defining "space ergonomics" are to name but a few. But these are issues we are already beginning to see evidence of. These hazards are imminent unless something is done to address them. However, with good foresight and dedicated analysis, the Human Factor and Biomechanics community should be able to fight off these hazards before they affect those who will do the exploring.

In this paper, a handful of issues are identified that will affect humans' physical health in long duration space travel and exploration. Now is the time to get to work on implementing appropriate solutions and discovering the rest of the problems before the crew start asking Mission Control where they packed the aspirin. 


\section{REFERENCES}

1. NASA (2004, Feb.). The Vision for Space Exploration. http://www.nasa.gov/pdf/55584main vision space e xploration-hi-res.pdf

2. Oberg, James (2004, Jan. 11 a). "Crew Finds 'Culprit' in Space Station Leak." MSNBC. HTML: http://msnbc.msn.com/id/3882962

3. Oberg, James (2004, Jan. 16 b). "Space Station Leak Caused by Crew, Experts Say." MSNBC. HTML: http://msnbc.msn.com/id/3969567

4. NASA (2001, May). Flight Projects - Operations Development Group - PERS Homepage. HTML: http://flightprojects. msfc.nasa.gov/fd36_pers.html

5. McCrory, J.L., et al. 1999 "Evaluation of a Treadmill With Vibration Isolation and Stabilization (TVIS) for
Use on the International Space Station" Journal of Applied Biomechanics, 1999, Vol. 15, pp. 292-302.

6. NASA. (1991). Space Station Freedom Man Systems Integration Standards (MSIS). June 1991, Houston, TX: NASA. (NASA-STD-3000, Vol. IV) (also available at msis.jsc.nasa.gov).

7. Mount, Francis et al. 2003 "Evaluation of Neutral Body Posture on Shuttle Mission STS-57 (SPACEHAB-1), NASA TM-2003-104805

\section{CONTACT}

George S. Hamilton

MS EV12, MSFC, AL 35812

George.s.hamilton@nasa.gov

256.544 .4963

Chris W. Adams, Raytheon

MS EV12, MSFC AL 35812

Chris.w.adams@msfc.naa.gov 256.544.0869 